\title{
Security Analysis of the GF-NLFSR Structure and Four-Cell Block Cipher
}

\author{
Wenling Wu, Lei Zhang, Liting Zhang and Wentao Zhang \\ State Key Laboratory of Information Security, Institute of Software, Chinese \\ Academy of Sciences, Beijing 100190, P.R.China
}

\begin{abstract}
The overall structure is one of the most important properties of block ciphers. At present, the most common structures include Feistel structure, SP structure, MISTY structure, L-M structure and Generalized Feistel structure. In [29], Choy et al. proposed a new structure called GF-NLFSR (Generalized Feistel-NonLinear Feedback Shift Register), and designed a new block cipher called Four-Cell which is based on the 4-cell GF-NLFSR. In this paper, we first study properties of the $n$ cell GF-NLFSR structure, and prove that for an $n$-cell GF-NLFSR, there exists an $\left(n^{2}+n-2\right)$ rounds impossible differential. Then we present an impossible differential attack on the full 25-round Four-Cell using this kind of 18-round impossible differential distinguisher together with differential cryptanalysis technique. The data complexity of our attack is $2^{111.5}$ and the time complexity is less than $2^{123.5}$ encryptions. In addition, we expect the attack to be more efficient when the relations between different round subkeys can be exploited by taking the key schedule algorithm into consideration.
\end{abstract}

Key words: GF-NLFSR structure, Four-Cell block cipher, Impossible differential cryptanalysis, Data complexity, Time complexity.

\section{Introduction}

The overall structure is one of the most important properties of block ciphers, and it plays important roles in the round number choice, software and hardware implementation performances and so on. At present, the most often used structures include Feistel structure, SP structure, MISTY structure, L-M structure and Generalized Feistel structure. Feistel structure was introduced by $\mathrm{H}$. Feistel in the design of Lucifer block cipher and later got famous since it was used in the design of DES. Feistel structure can transfer any function (usually called round function $F$ ) to a permutation. Now there are a lot of block ciphers employing the Feistel structure, such as Camellia, FEAL, GOST, LOKI, E2, Blowfish, RC5 and so on. The security of Feistel structure against differential and linear cryptanalysis was evaluated by many researchers, for example [1-3], and meanwhile there are many results such as [4-10] about the pseudorandomness of Feistel structure. Besides the Feistel structure, the other most often used structure is the SP structure, the well known block ciphers such as AES, Serpent 
and ARIA all employ the SP structure. In each round of the SP structure, first a layer of key-dependent inversive function named $S$ is applied to the input, and then applies a permutation or an inversive linear transformation named $P$. Hence the SP structure is very simple and clear, and $S$ is usually called the confusion layer which achieves confusion in the cipher and $P$ is usually referred to as the diffusion layer which diffuses efficiently. MISTY structure is another kind of important structures which was proposed by M. Matsui in [11], and it was used in the design of the block ciphers MISTY [12] and KASUMI [13]. There are many results about the security analysis of the MISTY structure such as in [14-17]. S. Vaudenay et al. named the structure of the block cipher IDEA as the L-M structure or Lai-Massey structure [18], and the FOX [19] cipher also employs a variant of the L-M structure. The generalized Feistel structure was first introduced by B. Schneier and J. Kelsey which can be considered as an unbalanced Feistel structure, and then many variants of generalized Feistel structure are proposed such as CAST-256-type Feistel structure, MARS-type Feistel structure, CLEFIA-type Feistel structure, SMS4-type Feistel structure and so on. All these kinds of generalized Feistel structures have similar advantages such as decryption - encryption similarity and the inverse of round function is not necessary in decryption. Furthermore, this can make the design of round function more simple and flexible. The security analysis of generalized Feistel structure is very important when they are used to design new block ciphers, and there are many results [25-28] about the security of different kinds of generalized Feistel structures against the differential and linear cryptanalysis and also their pseudorandomness.

In [29], Choy et al. proposed a new structure called GF-NLFSR (Generalized Feistel-NonLinear Feedback Shift Register). It can be considered as an $n$-cell extension of combining the MISTY structure and Generalized Unbalanced Feistel Network together. The security of the structure against many attacks such as differential, linear, impossible differential and integral cryptanalysis are also considered in [29]. For an $n$-cell GF-NLFSR, an upper bound for the differential and linear hull probabilities for any $n+1$ rounds are given, and a $2 n-1$ rounds impossible differential distinguisher and a $3 n-1$ rounds integral distinguisher on the $n$-cell GF-NLFSR are demonstrated. Furthermore, a new block cipher called Four-Cell which is based on the 4-cell GF-NLFSR was designed in [29]. The block and key size of Four-Cell are both 128-bit, and there are 25 rounds in total.

Impossible differential cryptanalysis [30] was first proposed by Biham, Biryukov and Shamir in 1999, and it was applied to analyze the Skipjack block cipher. Unlike differential cryptanalysis which exploits differentials with the highest possible probability, impossible differential cryptanalysis uses the differentials which hold with probability 0 , which can thus be called impossible differential. The impossible differentials can usually be built in a miss-in-the-middle manner. Recently, impossible differential cryptanalysis had received worldwide attention, and its application to block ciphers such as AES, Camellia and MISTY all achieved very good results [31-35]. 
In [29], Proposition 3 stated that for an $n$-cell GF-NLFSR, there exist at most $2 n-1$ rounds impossible differential distinguishers using the U-method proposed in [36]. However, we examine the property of $n$-cell GF-NLFSR structure and demonstrate that there exists a $\left(n^{2}+n-2\right)$ rounds impossible differential distinguisher. Then we present an impossible differential attack on the full 25round Four-Cell using this kind of 18-round impossible differential distinguisher together with differential cryptanalysis technique.

This paper is organized as follows. In Section 2, we give a brief description of the $n$-cell GF-NLFSR structure and Four-Cell block cipher. In Section 3, we describe some useful properties of the $n$-cell GF-NLFSR structure and the $\left(n^{2}+n-2\right)$ rounds impossible differential. Then in Section 4, we present our impossible differential attack on the full 25-round Four-Cell block cipher. Finally, in Section 5 we summarize this paper.

\section{The $n$-Cell GF-NLFSR Structure and Four-Cell Block Cipher}

\subsection{The $n$-Cell GF-NLFSR Structure}

In this section, we will give a brief description of the $n$-cell GF-NLFSR structure, and Fig. 1 below illustrates one round of GF-NLFSR.

For an $n$-cell GF-NLFSR structure, suppose the size of the internal sub-block is $m$-bit, and then we can denote the $m n$-bit input block as $\left(x_{1}, x_{2}, x_{3}, \ldots, x_{n}\right) \in$ $\left(\{0,1\}^{m}\right)^{n}$. If we denote the round subkey as $s k$, then the output of one round $n$-cell GF-NLFSR transformation is defined as follows.

$$
\begin{aligned}
& x_{2}=x_{2}, \\
& x_{3}=x_{3}, \\
& \cdots \\
& x_{n}=x_{n}, \\
& x_{n+1}=f\left(x_{1}, s k\right) \oplus x_{2} \oplus x_{3} \ldots \oplus x_{n},
\end{aligned}
$$

where the output block is denoted as $\left(x_{2}, x_{3}, \ldots, x_{n}, x_{n+1}\right) \in\left(\{0,1\}^{m}\right)^{n}$. Note here the symbol $\oplus$ is used to denote finite field addition (XOR) over $G F(2)^{m}$, and the function $f:\{0,1\}^{m} \times\{0,1\}^{k} \rightarrow\{0,1\}^{m}$ is the round function. Specifically, for each fixed round key sk, the round function $f(\cdot, s k):\{0,1\}^{m} \rightarrow\{0,1\}^{m}$ must be a permutation, or else the $n$-cell GF-NLFSR structure is not able to decrypt correctly. Therefore, in our later analysis, we will assume the round function $f$ is a permutation when the round key is fixed.

\section{$2.2 \quad$ Four-Cell Block Cipher}

The block and key size of Four-Cell are both 128-bit, and it uses the 4-cell GFNLFSR structure. Since the designers only give a rough suggestion for the key schedule algorithm, namely using a similar cipher with 26 rounds to generate the round keys needed. Hence in this paper, we will omit the key schedule and 


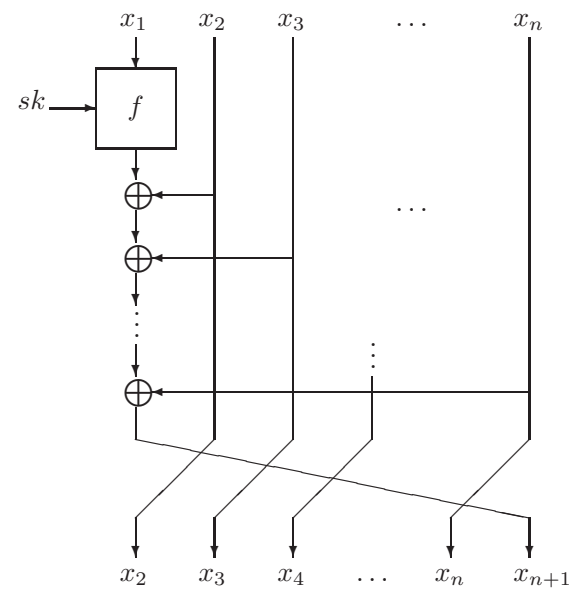

Fig. 1. One Round of $n$-Cell GF-NLFSR structure

just assume that the round keys are randomly chosen. The encryption algorithm of Four-Cell can be described briefly as follows.

Let the plaintext be denoted by $P=\left(x_{1}, x_{2}, x_{3}, x_{4}\right) \in\left(\{0,1\}^{32}\right)^{4}$, then after applying the full 25 rounds encryption, the 128-bit ciphertext can be denoted by $C$. Let $\left(x_{i}, x_{i+1}, x_{i+2}, x_{i+3}\right) \in\left(\{0,1\}^{32}\right)^{4}$ denote the input of the $i$-th round, then the output of the $i$-th round can be computed as follows.

$$
\begin{aligned}
& x_{i+1}=x_{i+1}, \\
& x_{i+2}=x_{i+2}, \\
& x_{i+3}=x_{i+3}, \\
& x_{i+4}=f_{i}\left(x_{i}, s k_{i}\right) \oplus x_{i+1} \oplus x_{i+2} \oplus x_{i+3} .
\end{aligned}
$$

For rounds $i=1,2, . ., 5$ and $i=21,22, . ., 25$, the round keys are denoted as $s k_{i} \in\{0,1\}^{32}$ and the round function is defined as $f_{i}\left(x_{i}, s k_{i}\right)=\operatorname{MDS}\left(S\left(x_{i} \oplus\right.\right.$ $\left.\left.s k_{i}\right)\right)$. For rounds $i=6,7, \ldots, 20$, the round keys are denoted as $s k_{i}=\left(s k_{i 0}, s k_{i 1}\right) \in$ $\left(\{0,1\}^{32}\right)^{2}$, and the round function is defined as $f_{i}\left(x_{i}, s k_{i}\right)=S\left(M D S\left(S\left(x_{i} \oplus\right.\right.\right.$ $\left.\left.\left.s k_{i 0}\right)\right) \oplus s k_{i 1}\right)$.

Here in each round function, $S:\left(\{0,1\}^{8}\right)^{4} \rightarrow\left(\{0,1\}^{8}\right)^{4}$ is four parallel $8 \times 8 s$-boxes, and the $s$-box is similar with the $s$-box used in the SubBytes operation in AES. The transformation $M D S:\left(\{0,1\}^{8}\right)^{4} \rightarrow\left(\{0,1\}^{8}\right)^{4}$ is a 4-byte to 4-byte maximal distance separable transform with optimal branch number 5 , and it is similar with the MixColumn operation in AES. In the end, the output after 25 rounds is XORed with a 128-bit post-whitening key $K_{26}=$ $\left(k_{26,1}, k_{26,2}, k_{26,3}, k_{26,4}\right)$ to get the ciphertext, namely $C=\left(x_{26} \oplus k_{26,1}, x_{27} \oplus\right.$ $\left.k_{26,2}, x_{28} \oplus k_{26,3}, x_{29} \oplus k_{26,4}\right)$. 


\section{Differential Property of the $n$-Cell GF-NLFSR Structure}

For the $n$-cell GF-NLFSR structure, we can express the $n m$-bit input as $n$ words which consists of $m$ bits each. Suppose we have a pair of plaintexts $X=\left(x_{1}, x_{2}, x_{3}, \ldots, x_{n}\right)$ and $X^{*}=\left(x_{1}^{*}, x_{2}^{*}, x_{3}^{*}, \ldots, x_{n}^{*}\right)$, and their difference is denoted by $\Delta X=\left(\Delta x_{1}, \Delta x_{2}, \ldots, \Delta x_{n}\right)$, where $\Delta x_{1}=x_{1} \oplus x_{1}^{*}, \ldots, \Delta x_{n}=x_{n} \oplus x_{n}^{*}$. Note the symbol 0 in the difference $\Delta X=\left(\Delta x_{1}, \Delta x_{2}, \Delta x_{3}, 0, \ldots, 0\right)$ means that the corresponding byte difference is zero.

Lemma 1. For the n-cell GF-NLFSR structure, there exists the following $n$ rounds differential characteristic whose probability is equal to 1.

$$
\left(\Delta x_{1}, \Delta x_{2}, \ldots, \Delta x_{i-1}, \Delta x_{i}, 0, \ldots, 0\right) \stackrel{n \text { rounds }}{\longrightarrow}\left(\Delta y_{1}, \Delta y_{2}, \ldots, \Delta y_{i}, \Delta y_{i+1}, 0, \ldots, 0\right) .
$$

We denote this kind of differential characteristic as $\Delta_{i}$, where $1 \leq i \leq n-1$, and these differential characteristics $\Delta_{i}$ satisfy the following two properties.

1. $\Delta y_{1} \oplus \Delta y_{2} \oplus \ldots \oplus \Delta y_{i} \oplus \Delta y_{i+1}=0$.

2. If $\Delta x_{i} \neq 0$, then $\Delta y_{i+1} \neq 0$.

Proof. Let the round function of Round $i$ be $f_{s k_{i}}\left(x_{i}\right)=f_{i}\left(x_{i}, s k_{i}\right)$. Then when the round key $s k_{i}$ is fixed, the round function $f_{s k_{i}}$ must be a permutation, or else one can not decrypt correctly for the $n$-cell GF-NLFSR structure. According to the structure of the $n$-cell GF-NLFSR, we can get the following equations which is illustrated in Table 1.

$$
\begin{aligned}
& \Delta y_{1}=f_{s k_{1}}\left(x_{1}\right) \oplus f_{s k_{1}}\left(x_{1} \oplus \Delta x_{1}\right) \oplus \Delta x_{2} \oplus \ldots \oplus \Delta x_{i} . \\
& \Delta y_{i}=f_{s k_{i}}\left(x_{i}\right) \oplus f_{s k_{i}}\left(x_{i} \oplus \Delta x_{i}\right) \oplus \Delta y_{1} \oplus \ldots \oplus \Delta y_{i-1} . \\
& \Delta y_{i+1}=\Delta y_{1} \oplus \ldots \oplus \Delta y_{i-1} \oplus \Delta y_{i} .
\end{aligned}
$$

Table 1. The $\mathrm{n}$ rounds differential characteristic of the $n$-cell GF-NLFSR structure

\begin{tabular}{|c||c|c|c|c|c|c|c|c|}
\hline Round $\backslash$ Diff. & $\Delta x_{1}$ & $\Delta x_{2}$ & $\ldots$ & $\Delta x_{i-1}$ & $\Delta x_{i}$ & 0 & $\ldots$ & 0 \\
\hline 1 & $\Delta x_{2}$ & $\Delta x_{3}$ & $\ldots$ & $\Delta x_{i}$ & 0 & $\ldots$ & 0 & $\Delta y_{1}$ \\
\hline$\vdots$ & $\vdots$ & $\vdots$ & $\vdots$ & $\vdots$ & $\vdots$ & $\vdots$ & $\vdots$ & $\vdots$ \\
\hline$i-1$ & $\Delta x_{i}$ & 0 & $\ldots$ & 0 & 0 & $\Delta y_{1}$ & $\ldots$ & $\Delta y_{i-1}$ \\
\hline$i$ & 0 & $\ldots$ & 0 & 0 & $\Delta y_{1}$ & $\ldots$ & $\Delta y_{i-1}$ & $\Delta y_{i}$ \\
\hline$i+1$ & 0 & $\ldots$ & 0 & $\Delta y_{1}$ & $\ldots$ & $\Delta y_{i-1}$ & $\Delta y_{i}$ & $\Delta y_{i+1}$ \\
\hline$i+2$ & 0 & $\ldots$ & $\Delta y_{1}$ & $\ldots$ & $\Delta y_{i-1}$ & $\Delta y_{i}$ & $\Delta y_{i+1}$ & 0 \\
\hline$\vdots$ & $\vdots$ & $\vdots$ & $\vdots$ & $\vdots$ & $\vdots$ & $\vdots$ & $\vdots$ & $\vdots$ \\
\hline$n$ & $\Delta y_{1}$ & $\Delta y_{2}$ & $\ldots$ & $\Delta y_{i}$ & $\Delta y_{i+1}$ & 0 & $\ldots$ & 0 \\
\hline
\end{tabular}


According to Equ. (3), the following one round differential characteristic will hold with probability 1 .

$$
\left(0, \ldots, 0, \Delta y_{1}, \ldots, \Delta y_{i-1}, \Delta y_{i}, \Delta y_{i+1}\right) \stackrel{1 \text { round }}{\longrightarrow}\left(0, \ldots, 0, \Delta y_{1}, \ldots, \Delta y_{i}, \Delta y_{i+1}, 0\right) .
$$

Similarly, we can know that all the differential characteristics from Round $(i+2)$ to Round $n$ in Table 1 hold with probability 1 . Therefore, for the $n$-cell GFNLFSR structure, there exists the following $n$ rounds differential characteristic and its probability is equal to 1 .

$$
\left(\Delta x_{1}, \Delta x_{2}, \ldots, \Delta x_{i-1}, \Delta x_{i}, 0, \ldots, 0\right) \stackrel{n \text { rounds }}{\longrightarrow}\left(\Delta y_{1}, \Delta y_{2}, \ldots, \Delta y_{i}, \Delta y_{i+1}, 0, \ldots, 0\right)
$$

Then according to Equ. (3), we can easily get the first property, i.e. $\Delta y_{1} \oplus$ $\Delta y_{2} \oplus \ldots \oplus \Delta y_{i} \oplus \Delta y_{i+1}=0$. Therefore, in the following we only need to prove the second property.

According to Equ. (2) and Equ. (3), we can get the following equation.

$$
\Delta y_{i+1}=\Delta y_{1} \oplus \ldots \oplus \Delta y_{i-1} \oplus \Delta y_{i}=f_{s k_{i}}\left(x_{i}\right) \oplus f_{s k_{i}}\left(x_{i} \oplus \Delta x_{i}\right) .
$$

When $\Delta x_{i} \neq 0$, we can conclude that $f_{s k_{i}}\left(x_{i}\right) \oplus f_{s k_{i}}\left(x_{i} \oplus \Delta x_{i}\right) \neq 0$ since the function $f_{s k_{i}}$ is a permutation. Therefore, we get the second property, namely if $\Delta x_{i} \neq 0$, then $\Delta y_{i+1} \neq 0$.

Lemma 2. For the inverse of the $n$-cell GF-NLFSR structure which is denoted as the $n$-cell GF-NLFSR ${ }^{-1}$ structure, there exists the following $(2 n-2)$ rounds differential characteristic whose probability is equal to 1.

$$
(\beta, \beta, 0, \ldots, 0) \stackrel{2 n-2 \text { rounds }}{\longrightarrow}\left(?, \ldots, ?, b_{2}, b_{1}, 0\right) .
$$

We denote this kind of differential characteristic as $\Delta_{\beta}^{-1}$, where the symbol ? denotes an unknown difference and $\beta, b_{2}, b_{1}$ denote non-zero differences.

Proof. According to the structure of the $n$-cell GF-NLFSR ${ }^{-1}$, we can get the following one round differential characteristic which holds with probability 1 , and this kind of differential is illustrated in Table 2.

$$
(\beta, \beta, 0, \ldots, 0) \stackrel{1 \text { round }}{\longrightarrow}(0, \beta, \beta, 0, \ldots, 0) .
$$

Similarly, all the differential characteristics from the Round 2 to Round $(n-1)$ in Table 2 all hold with probability 1 . Then in the Round $n$, if we denote the round function as $g_{n}$, then $b_{1}=g_{n}(z) \oplus g_{n}(z \oplus \beta)$. Because the difference $\beta$ is non-zero and the function $g_{n}$ is a permutation, we can conclude that $b_{1} \neq 0$. Similarly, in the $(n+1)$-th round we have $b_{2}=g_{n+1}(w) \oplus g_{n+1}\left(w \oplus b_{1}\right)$, and thus we can conclude that $b_{2} \neq 0$ since $b_{1} \neq 0$.

Finally, according to the property of the $n$-cell GF-NLFSR ${ }^{-1}$ structure, the differential characteristics from Round $(n+2)$ to Round $(2 n-2)$ in Table 2 all hold with probability 1 . Therefore, for the $n$-cell GF-NLFSR ${ }^{-1}$ structure, there exists the following differential characteristic whose probability is equal to 1 .

$$
(\beta, \beta, 0, \ldots, 0) \stackrel{2 n-2 \text { rounds }}{\longrightarrow}\left(?, \ldots, ?, b_{2}, b_{1}, 0\right) .
$$


Table 2. The $(2 n-2)$ rounds differential of the $n$-cell GF-NLFSR ${ }^{-1}$ structure

\begin{tabular}{|c||c|c|c|c|c|c|}
\hline Round $\backslash$ Diff. & $\beta$ & $\beta$ & 0 & 0 & $\ldots$ & 0 \\
\hline 1 & 0 & $\beta$ & $\beta$ & 0 & $\ldots$ & 0 \\
\hline$\vdots$ & $\vdots$ & $\vdots$ & $\vdots$ & $\vdots$ & $\vdots$ & $\vdots$ \\
\hline$n-2$ & 0 & 0 & $\ldots$ & 0 & $\beta$ & $\beta$ \\
\hline$n-1$ & 0 & 0 & $\ldots$ & 0 & 0 & $\beta$ \\
\hline$n$ & $b_{1}$ & 0 & 0 & $\ldots$ & 0 & 0 \\
\hline$n+1$ & $b_{2}$ & $b_{1}$ & 0 & $\ldots$ & 0 & 0 \\
\hline$\vdots$ & $\vdots$ & $\vdots$ & $\vdots$ & $\vdots$ & $\vdots$ & $\vdots$ \\
\hline $2 n-2$ & $?$ & $\ldots$ & $?$ & $b_{2}$ & $b_{1}$ & 0 \\
\hline
\end{tabular}

Theorem 1. For the n-cell GF-NLFSR structure, there exists the following kind of $\left(n^{2}+n-2\right)$ rounds impossible differential where $\alpha$ and $\beta$ are non-zero differences.

$$
(\alpha, 0, \ldots, 0) \stackrel{n^{2}+n-2 \text { rounds }}{\longrightarrow}(\beta, \beta, 0, \ldots, 0) .
$$

Proof. This kind of $\left(n^{2}+n-2\right)$ rounds impossible differential is constructed using the miss-in-the-middle technique. First we construct an $n(n-1)$ rounds differential characteristic of the encryption direction and an $(2 n-2)$ rounds differential characteristic of the decryption direction whose probabilities are both equal to 1 . Then if these two differential characteristics contradict each other in the middle, we get the $\left(n^{2}+n-2\right)$ rounds impossible differential. In Table 3 we illustrate this kind of impossible differential in detail.

When we choose the input difference as $(\alpha, 0, \ldots, 0)$, we can construct an $n(n-1)$ rounds differential with probability 1 as follows. First of all, based on Lemma 1 , we can construct the following $n$ rounds differential $\Delta_{1}$ whose probability is equal to 1 .

$$
(\alpha, 0, \ldots, 0) \stackrel{n \text { rounds }}{\longrightarrow}\left(\Delta x_{1}^{2}, \Delta x_{2}^{2}, 0, \ldots, 0\right) .
$$

Since the input difference $\alpha$ is non-zero, according to property 1 and 2 of Lemma 1 , we know that $\Delta x_{2}^{2}$ is also non-zero and $\Delta x_{1}^{2} \oplus \Delta x_{2}^{2}=0$.

Then, we start with the input difference of $\left(\Delta x_{1}^{2}, \Delta x_{2}^{2}, 0, \ldots, 0\right)$, and according to Lemma 1 , we can construct again an $n$ rounds differential $\Delta_{2}$ whose probability is 1 as follows.

$$
\left(\Delta x_{1}^{2}, \Delta x_{2}^{2}, 0, \ldots, 0\right) \stackrel{n \text { rounds }}{\longrightarrow}\left(\Delta x_{1}^{3}, \Delta x_{2}^{3}, \Delta x_{3}^{3}, 0, \ldots, 0\right) .
$$

Since $\Delta x_{2}^{2}$ is non-zero, we know that $\Delta x_{3}^{3} \neq 0$ and $\Delta x_{1}^{3} \oplus \Delta x_{2}^{3} \oplus \Delta x_{3}^{3}=0$. Similarly, we can construct the $i$-th $(3 \leq i \leq n-1) n$ rounds differential $\Delta_{i}$ in turn. In the end, the $(n-1)$-th $\mathrm{n}$ rounds differential $\Delta_{n-1}$ is as follows, and we can conclude that $\Delta x_{n}^{n} \neq 0$ and $\Delta x_{1}^{n} \oplus \Delta x_{2}^{n} \oplus \Delta x_{3}^{n} \oplus \ldots \oplus \Delta x_{n}^{n}=0$.

$$
\left(\Delta x_{1}^{n-1}, \Delta x_{2}^{n-1}, \ldots, \Delta x_{n-1}^{n-1}, 0\right) \stackrel{n \text { rounds }}{\longrightarrow}\left(\Delta x_{1}^{n}, \Delta x_{2}^{n}, \Delta x_{3}^{n}, \ldots, \Delta x_{n}^{n}\right) .
$$


By concatenating the above differentials together, we can get the following $n(n-1)$ rounds differential whose probability is equal to 1 and $\Delta x_{n}^{n}$ is non-zero.

$$
(\alpha, 0, \ldots, 0) \stackrel{n(n-1) \text { rounds }}{\longrightarrow}\left(\Delta x_{1}^{n}, \Delta x_{2}^{n}, \Delta x_{3}^{n}, \ldots, \Delta x_{n}^{n}\right) .
$$

Table 3. The $\left(n^{2}+n-2\right)$ rounds impossible differential of $n$-cell GF-NLFSR structure

\begin{tabular}{|c||c|c|c|c|c|c|}
\hline Round $\backslash$ Diff. & $\alpha$ & 0 & 0 & $\ldots$ & 0 & 0 \\
\hline 1 & 0 & 0 & 0 & $\ldots$ & 0 & $\Delta x_{1}^{2}$ \\
\hline 2 & 0 & 0 & $\ldots$ & 0 & $\Delta x_{1}^{2}$ & $\Delta x_{2}^{2}$ \\
\hline 3 & 0 & $\ldots$ & 0 & $\Delta x_{1}^{2}$ & $\Delta x_{2}^{2}$ & 0 \\
\hline$\vdots$ & $\vdots$ & $\vdots$ & $\vdots$ & $\vdots$ & $\vdots$ & $\vdots$ \\
\hline$n$ & $\Delta x_{1}^{2}$ & $\Delta x_{2}^{2}$ & 0 & $\ldots$ & 0 & 0 \\
\hline$n+1$ & $\Delta x_{2}^{2}$ & 0 & 0 & $\ldots$ & 0 & $\Delta x_{1}^{3}$ \\
\hline$n+2$ & 0 & 0 & $\ldots$ & 0 & $\Delta x_{1}^{3}$ & $\Delta x_{2}^{3}$ \\
\hline$n+3$ & 0 & $\ldots$ & 0 & $\Delta x_{1}^{3}$ & $\Delta x_{2}^{3}$ & $\Delta x_{3}^{3}$ \\
\hline$\vdots$ & $\vdots$ & $\vdots$ & $\vdots$ & $\vdots$ & $\vdots$ & $\vdots$ \\
\hline $2 n$ & $\Delta x_{1}^{3}$ & $\Delta x_{2}^{3}$ & $\Delta x_{3}^{3}$ & 0 & $\ldots$ & 0 \\
\hline$\vdots$ & $\vdots$ & $\vdots$ & $\vdots$ & $\vdots$ & $\vdots$ & $\vdots$ \\
\hline$n(n-2)$ & $\Delta x_{1}^{n-1}$ & $\Delta x_{2}^{n-1}$ & $\Delta x_{3}^{n-1}$ & $\ldots$ & $\Delta x_{n-1}^{n-1}$ & 0 \\
\hline$\vdots$ & $\vdots$ & $\vdots$ & $\vdots$ & $\vdots$ & $\vdots$ & $\vdots$ \\
\hline$n(n-1)$ & $\Delta x_{1}^{n}$ & $\Delta x_{2}^{n}$ & $\Delta x_{3}^{n}$ & $\ldots$ & $\Delta x_{n}^{n}$ & $\Delta x_{n}^{n}$ \\
\hline
\end{tabular}

\begin{tabular}{|c||c|c|c|c|c|c|}
\hline & $?$ & $\ldots$ & $?$ & $b_{2}$ & $b_{1}$ & 0 \\
\hline$n(n-1)+1$ & $?$ & $\ldots$ & $b_{2}$ & $b_{1}$ & 0 & 0 \\
\hline$\vdots$ & $\vdots$ & $\vdots$ & $\vdots$ & $\vdots$ & $\vdots$ & $\vdots$ \\
\hline$n^{2}-3$ & $b_{2}$ & $b_{1}$ & 0 & 0 & $\ldots$ & 0 \\
\hline$n^{2}-2$ & $b_{1}$ & 0 & 0 & $\ldots$ & 0 & 0 \\
\hline$n^{2}-1$ & 0 & 0 & $\ldots$ & 0 & 0 & $\beta$ \\
\hline$n^{2}$ & 0 & 0 & $\ldots$ & 0 & $\beta$ & $\beta$ \\
\hline$\vdots$ & $\vdots$ & $\vdots$ & $\vdots$ & $\vdots$ & $\vdots$ & $\vdots$ \\
\hline$n^{2}+n-3$ & 0 & $\beta$ & $\beta$ & 0 & $\ldots$ & 0 \\
\hline$n^{2}+n-2$ & $\beta$ & $\beta$ & 0 & 0 & $\ldots$ & 0 \\
\hline
\end{tabular}

In the decryption direction, considering the inverse structure $n$-cell GF$\mathrm{NLFSR}^{-1}$, we get the following $2 n-2$ rounds differential characteristic with probability 1 according to Lemma 2 .

$$
(\beta, \beta, 0, \ldots, 0) \stackrel{2 n-2 \text { rounds }}{\longrightarrow}\left(?, \ldots, ?, b_{2}, b_{1}, 0\right)
$$


If we concatenate the above $n(n-1)$ rounds differential of the encryption direction and the $(2 n-2)$ rounds differential of the decryption direction together, we can construct the following $\left(n^{2}+n-2\right)$ rounds impossible differential since they contradict each other at $\Delta x_{n}^{n}$.

$$
(\alpha, 0,0, \ldots, 0) \stackrel{\left(n^{2}+n-2\right) \text { rounds }}{\nrightarrow}(\beta, \beta, 0, \ldots, 0) .
$$

\section{Security Analysis of Four-Cell Block Cipher}

According to Theorem 1, for Four-Cell block cipher which employs the 4-cell GFNLFSR structure, there exists an 18 rounds impossible differential as follows.

$$
(\alpha, 0,0,0) \stackrel{18 \text { rounds }}{\nrightarrow}(\beta, \beta, 0,0)
$$

By setting the 18-round impossible differential distinguisher in the middle rounds, we can present an impossible differential attack on the full 25-round Four-Cell by analyzing the first 4 rounds before and the last 3 rounds after the distinguisher. Note the round functions of the first 5 rounds and the last 5 rounds are all defined as $f_{i}\left(x_{i}, s k_{i}\right)=M D S\left(S\left(x_{i} \oplus s k_{i}\right)\right)$. The attack procedure is illustrated in Fig. 2 in Appendix A.

Let the plaintext be $X=\left(x_{1}, x_{2}, x_{3}, x_{4}\right) \in\left(\{0,1\}^{32}\right)^{4}$, then the intermediate state after 3 rounds and 4 rounds encryption can be denoted as $\left(x_{4}, x_{5}, x_{6}, x_{7}\right)$ and $\left(x_{5}, x_{6}, x_{7}, x_{8}\right)$ respectively. Furthermore, the intermediate state after 22 rounds encryption can be denoted as $\left(x_{23}, x_{24}, x_{25}, x_{26}\right)$ and the 128-bit ciphertext should be $C=\left(c_{1}, c_{2}, c_{3}, c_{4}\right)=\left(x_{26} \oplus k_{26,1}, x_{27} \oplus k_{26,2}, x_{28} \oplus k_{26,3}, x_{29} \oplus k_{26,4}\right)$. Suppose we choose another plaintext $X^{*}=\left(x_{1}^{*}, x_{2}^{*}, x_{3}^{*}, x_{4}^{*}\right) \in\left(\{0,1\}^{32}\right)^{4}$, and the plaintext difference can be denoted as $\Delta x_{i}=x_{i} \oplus x_{i}^{*}$.

Then for the last three rounds of Four-Cell, we have the following equations.

$$
\begin{aligned}
& x_{27}=M D S\left(S\left(x_{23} \oplus s k_{23}\right)\right) \oplus x_{24} \oplus x_{25} \oplus x_{26}, \\
& x_{28}=M D S\left(S\left(x_{24} \oplus s k_{24}\right)\right) \oplus x_{25} \oplus x_{26} \oplus x_{27}, \\
& x_{29}=M D S\left(S\left(x_{25} \oplus s k_{25}\right)\right) \oplus x_{26} \oplus x_{27} \oplus x_{28} .
\end{aligned}
$$

If we denote $r k_{25}=k_{26,1} \oplus k_{26,2} \oplus k_{26,3} \oplus k_{26,4}$, then the input of the Sbox layer for Round 25 can be computed as follows.

$$
y_{25}=S^{-1}\left(M D S^{-1}\left(c_{1} \oplus c_{2} \oplus c_{3} \oplus c_{4} \oplus r k_{25}\right)\right)=x_{25} \oplus s k_{25} .
$$

Similarly, we can denote $r k_{24}=s k_{25} \oplus k_{26,1} \oplus k_{26,2} \oplus k_{26,3}$, and compute the input of the Sbox layer for Round 24 as follows.

$$
y_{24}=S^{-1}\left(M D S^{-1}\left(c_{1} \oplus c_{2} \oplus c_{3} \oplus y_{25} \oplus r k_{24}\right)\right)=x_{24} \oplus s k_{24}
$$


Finally, for Round 23 the output of the round function is $c_{1} \oplus c_{2} \oplus y_{24} \oplus y_{25} \oplus$ $s k_{25} \oplus s k_{24} \oplus k_{26,1} \oplus k_{26,2}$. If we denote $r k_{23}=s k_{24} \oplus s k_{25} \oplus k_{26,1} \oplus k_{26,2}$, then the input of the round function can be computed in a similar way.

$$
y_{23}=S^{-1}\left(M D S^{-1}\left(c_{1} \oplus c_{2} \oplus y_{24} \oplus y_{25} \oplus r k_{23}\right)\right)=x_{23} \oplus s k_{23} .
$$

Therefore, considering that $\Delta x_{23}=\Delta y_{23}, \Delta x_{24}=\Delta y_{24}, \Delta x_{25}=\Delta y_{25}$ and $\Delta x_{26}=\Delta c_{1}$, we can obtain the values of $\left(\Delta x_{23}, \Delta x_{24}, \Delta x_{25}, \Delta x_{26}\right)$ by just computing the values of $y_{25}, y_{24}$ and $y_{23}$ for a pair of ciphertexts $C=\left(c_{1}, c_{2}, c_{3}, c_{4}\right)$ and $C^{*}=\left(c_{1}^{*}, c_{2}^{*}, c_{3}^{*}, c_{4}^{*}\right)$.

For the first four rounds of Four-Cell, if we choose the plaintext difference as $\left(\Delta x_{1}, \Delta x_{2}, \Delta x_{3}, \Delta x_{4}\right)=(0,0,0, \alpha)$, then we can get the following equations.

$$
\begin{aligned}
& \Delta x_{5}=\alpha, \\
& \Delta x_{6}=0, \\
& \Delta x_{7}=0, \\
& \Delta x_{8}=M S D\left(S\left(x_{4} \oplus s k_{4}\right)\right) \oplus M S D\left(S\left(x_{4} \oplus \alpha \oplus s k_{4}\right)\right) \oplus \alpha .
\end{aligned}
$$

Here, $\Delta x_{8}=0$ holds if and only if $S\left(x_{4} \oplus s k_{4}\right) \oplus S\left(x_{4} \oplus \alpha \oplus s k_{4}\right)=M D S^{-1}(\alpha)$. Because the branch number of $M D S$ is 5 , there is at most one passive byte of $\alpha$. For simplicity, we can assume the last byte of $\alpha$ is passive.

Let $\alpha=\left(\alpha_{1}, \alpha_{2}, \alpha_{3}, \alpha_{4}\right) \in\left(\{0,1\}^{8}\right)^{4}$ and $\beta=\left(\beta_{1}, \beta_{2}, \beta_{3}, \beta_{4}\right) \in\left(\{0,1\}^{8}\right)^{4}$, and then we will use the symbol $\alpha \stackrel{S}{\longleftrightarrow} \beta$ to express that there exists $x_{i}=$ $\left(x_{i 1}, x_{i 2}, x_{i 3}, x_{i 4}\right) \in\left(\{0,1\}^{8}\right)^{4}$ such that $S\left(x_{i}\right) \oplus S\left(x_{i} \oplus \alpha\right)=\beta$.

Therefore, we can choose a set $A$ which is defined as follows.

$$
\mathrm{A}=\left\{\alpha=\left(\alpha_{1}, \alpha_{2}, \alpha_{3}, 0\right) \in\left(\{0,1\}^{8}\right)^{4} \mid \alpha \stackrel{S}{\longleftrightarrow} M D S^{-1}(\alpha)\right\} .
$$

Note here the necessary condition for $\alpha \stackrel{S}{\longleftrightarrow} M D S^{-1}(\alpha)$ is that $\alpha_{1}, \alpha_{2}$, and $\alpha_{3}$ should satisfy a linear relation (e.g. for the MDS used in AES, the linear relation is $\left.0 b \cdot \alpha_{1} \oplus 0 d \cdot \alpha_{2} \oplus 09 \cdot \alpha_{3}=0\right)$. Furthermore, for the Sbox of Four-Cell, the probability of $\alpha_{i} \stackrel{S}{\longrightarrow} \beta_{i}$ holds is about $2^{-1}$ for $\forall \beta_{i} \in\{0,1\}^{8}$. Therefore, the set $A$ contains about $|\mathrm{A}| \approx\left(2^{8}-1\right) \times\left(2^{8}-1\right) \times 2^{-1} \times 2^{-1} \times 2^{-1} \approx 2^{13}$ possible values. We also test this estimation using computer program, and with the same MDS and the Sbox used in AES, our searching result shows that the set $A$ contains $7965 \approx 2^{12.96}$ possible values of $\alpha$ which is very close to the theory estimation.

After analyzing the first four rounds and the last three rounds of Four-Cell, we can set the 18-round impossible differential at Round 5 to Round 22 and apply an impossible differential attack on the full 25-round Four-Cell. The attack procedure consists of three steps, and we will utilize impossible differential attack technique together with some properties of the structure.

The first step of the attack is data collection. We first choose appropriate plaintext structures defined as follows.

$$
S_{P}=\left\{\left(a_{1}, a_{2}, a_{3}, x_{4}\right)\right\},
$$

where $a_{1}, a_{2}, a_{3}$ are 32-bit constants and the last byte of $x_{4}$ is also an 8-bit constants, namely $x_{4}=\left(x_{41}, x_{42}, x_{43}, a_{44}\right) \in\left(\{0,1\}^{8}\right)^{4}, x_{4 j} \in\{0,1\}^{8}$. Therefore, 
each structure contains $2^{24}$ plaintexts and they can construct about $2^{24} \times 2^{13} / 2 \approx$ $2^{36}$ useful pairs whose plaintext differences satisfy the conditions listed above.

The second step of the attack is data filtering, in which we will discard all the useless pairs which do not satisfy the corresponding ciphertext difference. Note the output difference after the impossible differential distinguisher is $\left(\Delta x_{23}, \Delta x_{24}, \Delta x_{25}, \Delta x_{26}\right)=(\beta, \beta, 0,0)$, and according to the structure of FourCell, the ciphertext difference $\left(\Delta c_{1}, \Delta c_{2}, \Delta c_{3}, \Delta c_{4}\right)=\left(\Delta x_{26}, \Delta x_{27}, \Delta x_{28}, \Delta x_{29}\right)$ should satisfy the following two conditions.

$$
\begin{aligned}
& \Delta c_{1}=0, \\
& \Delta c_{1} \oplus \Delta c_{2} \oplus \Delta c_{3} \oplus \Delta c_{4}=0 .
\end{aligned}
$$

Therefore, the probability of a pair remains after this filtering is about $2^{-64}$.

The third step of the attack is key recovery. First of all, for each guess of $\left(s k_{41}, s k_{42}, s k_{43}\right)$ we can partially encrypt Round 4 to check if a pair satisfies the distinguisher. Note for each plaintext pair $X=\left(x_{1}, x_{2}, x_{3}, x_{4}\right)$ and $X^{*}=$ $\left(x_{1}^{*}, x_{2}^{*}, x_{3}^{*}, x_{4}^{*}\right)$, a useful pair must satisfy that the output difference of the round function in Round 4 equals to the input difference $x_{4} \oplus x_{4}^{*}$. Therefore, based on this property we can discard some useless pairs to reduce the complexity in the following steps, and the probability of a pair remains after this filtering is about $2^{-21}$. Then for all the remained pairs, we guess the values of $r k_{25}$ and $r k_{24}$ to decrypt Round 25 and Round 24 respectively. At last we recover the value of $r k_{23}$ by differential techniques. Then we can discard all the wrong subkey guesses using the impossible differential sieving techniques.

In the following, we will describe the attack procedure in detail.

1. Data Collection: Choose $2^{m}$ structures and each structure is constructed as follows:

$$
\begin{aligned}
& x_{1}=a_{1}, \\
& x_{2}=a_{2}, \\
& x_{3}=a_{3}, \\
& x_{4}=\left(x_{41}, x_{42}, x_{43}, a_{44}\right),
\end{aligned}
$$

where $\left(a_{1}, a_{2}, a_{3}\right)$ are 32 -bit constants, $a_{44}$ is 8 -bit constant and the 3 bytes $\left(x_{41}, x_{42}, x_{43}\right)$ take all the possible values of $\left(\{0,1\}^{8}\right)^{3}$. Then each structure contains $2^{24}$ plaintexts, which can generate about $2^{24} \cdot 2^{13} / 2=2^{36}$ plaintext pairs. Therefore, $2^{m}$ structures can generate about $2^{m+36}$ plaintext pairs.

2. Data Filtering: According to the property of ciphertext difference, for a useful pair the difference $\left(\Delta c_{1}, \Delta c_{2}, \Delta c_{3}, \Delta c_{4}\right)$ should satisfy the following conditions.

$$
\begin{aligned}
& \Delta c_{1}=0, \\
& \Delta c_{1} \oplus \Delta c_{2} \oplus \Delta c_{3} \oplus \Delta c_{4}=0 .
\end{aligned}
$$

Therefore, after this test the expected number of remaining pairs is about $2^{m+36} \cdot 2^{-64}=2^{m-28}$.

3 . For each guess of the 24-bit subkey $\left(s k_{41}, s k_{42}, s k_{43}\right)$, proceed as follows:

(a) List all the possible values of $r k_{23}$ as a table $L$. 
(b) For each of the remaining plaintext pair $X=\left(x_{1}, x_{2}, x_{3}, x_{4}\right)$ and $X^{*}=$ $\left(x_{1}^{*}, x_{2}^{*}, x_{3}^{*}, x_{4}^{*}\right)$, partially encrypt Round 4 to compute the following values respectively.

$$
\begin{aligned}
\gamma=\left(s\left(x_{41} \oplus s k_{41}\right) \oplus s\left(x_{41}^{*} \oplus s k_{41}\right),\right. & s\left(x_{42} \oplus s k_{42}\right) \oplus s\left(x_{42}^{*} \oplus s k_{42}\right), \\
s\left(x_{43} \oplus s k_{43}\right) \oplus s\left(x_{43}^{*} \oplus s k_{43}\right), & 0) \\
\lambda= & M D S^{-1}\left(x_{4} \oplus x_{4}^{*}\right) .
\end{aligned}
$$

Then check if $\gamma=\lambda$ holds, and if this is not the case, discard the corresponding plaintext pair. After this test, there remains about $2^{m-28}$. $2^{-21}=2^{m-49}$ plaintext pairs.

(c) Guess the value of $r k_{25}=k_{26,1} \oplus k_{26,2} \oplus k_{26,3} \oplus k_{26,4}$, and for each of the remaining pair, whose ciphertexts are denoted as $\left(c_{1}, c_{2}, c_{3}, c_{4}\right)$ and $\left(c_{1}^{*}, c_{2}^{*}, c_{3}^{*}, c_{4}^{*}\right)$ respectively, do as follows.

i. Compute the value of $y_{25}$ as follows.

$$
y_{25}=S^{-1}\left(M D S^{-1}\left(c_{1} \oplus c_{2} \oplus c_{3} \oplus c_{4} \oplus r k_{25}\right)\right) .
$$

Note for the remaining pairs we have $\Delta y_{25}=0$, and $y_{25}^{*}=S^{-1}\left(M D S^{-1}\left(c_{1}^{*} \oplus\right.\right.$ $\left.\left.c_{2}^{*} \oplus c_{3}^{*} \oplus c_{4}^{*} \oplus r k_{25}\right)\right)=y_{25}$.

ii. For each guess of the value $r k_{24}=s k_{25} \oplus k_{26,1} \oplus k_{26,2} \oplus k_{26,3}$, continue to compute the values of $y_{24}$ and $y_{24}^{*}$ as follows.

$$
\begin{aligned}
& y_{24}=S^{-1}\left(M D S^{-1}\left(c_{1} \oplus c_{2} \oplus c_{3} \oplus y_{25} \oplus r k_{24}\right)\right), \\
& y_{24}^{*}=S^{-1}\left(M D S^{-1}\left(c_{1}^{*} \oplus c_{2}^{*} \oplus c_{3}^{*} \oplus y_{25}^{*} \oplus r k_{24}\right)\right) .
\end{aligned}
$$

iii. If we denote the decryption function of Round 23 as $g\left(z, r k_{23}\right)=$ $S^{-1}\left(M D S^{-1}\left(z \oplus r k_{23}\right)\right)$, then for each remaining pair the inputs of $g$ are $c_{1} \oplus c_{2} \oplus y_{24} \oplus y_{25}$ and $c_{1}^{*} \oplus c_{2}^{*} \oplus y_{24}^{*} \oplus y_{25}^{*}$ respectively, and the output difference of $g$ should be $y_{24} \oplus y_{24}^{*}$. Therefore, by making use of the difference distribution table of Sbox we can compute the corresponding value of subkey $r k_{23}$. Discard it from the table $L$.

iv. If the table $L$ is not empty after analyzing all the remaining pairs, we can output the value of $r k_{23}$ remained in table $L$ together with the corresponding guess of $\left(s k_{41}, s k_{42}, s k_{43}\right), r k_{25}$ and $r k_{24}$ as the correct subkey.

If we choose $m=2^{87.5}$, then the number of useful pairs remained after the data filtering in Step 2 is about $2^{59.5}$. Hence there remains about $2^{38.5}$ pairs after the test of Step 3.b). In Step 3.c), according to the difference distribution table of Sbox, each pair can discard about one candidate of $r k_{23}$. Since there are $2^{32}$ possible values of $r k_{23}$ in table $L$, then after analyzing all the $2^{38.5}$ remaining pairs, the probability of a subkey guess of $r k_{23}$ still remains in $L$ is about $\left(1-2^{-32}\right)^{2^{38.5}} \approx e^{-2^{6.5}}$. Therefore, in Step 3.c.iv) the probability of a wrong subkey guess still remains after all the tests is about $2^{120} \times e^{-2^{6.5}}<2^{-11}$, and this means that only the correct subkey will be output.

The data and time complexities of the attack can be estimated as follows. First of all, we choose $2^{87.5}$ structures which contains $2^{24}$ plaintexts each, and 
thus the data complexity of the attack is about $2^{24} \times 2^{87.5}=2^{111.5}$ chosen plaintexts.

The time complexity of each step can be estimated roughly as follows. In Step 1, we need about $2^{111.5}$ encryptions. In Step 2 we have to check if the pair satisfies the ciphertext difference for all the $2^{123.5}$ pairs. Note the time needed for filtering is rather small which can be estimated as $2^{-3}$-round encryption. Therefore, the time complexity of Step 2 is about $2^{123.5} \times \frac{1}{25} \times 2^{-3}<2^{115.9}$ encryptions. In Step 3.b), we need to encrypt one round for each pair, which means that the time complexity is about $2^{24} \times 2^{59.5} / 25>2^{78.9}$ encryptions. Similarly, the time complexities of Step 3.c.i) and Step 3.c.ii) are $2^{24} \times 2^{32} \times 2^{38.5} \times 1 / 25<2^{89.9}$ encryptions and $2^{24} \times 2^{32} \times 2^{32} \times 2^{38.5} \times 2 / 25<2^{122.9}$ encryptions respectively. In Step 3.c.iii), the operation to recover subkey $r k_{23}$ from the difference distribution table of Sbox is rather simple and can be estimated as 1-round encryption. Then the time complexity of this step is about $2^{24} \times 2^{32} \times 2^{32} \times 2^{38.5} \times 1 / 25<2^{121.9}$ encryptions. Therefore, the total time complexity of the attack is less than $2^{123.5}$ encryptions.

\section{Conclusion}

In [29], Choy et al proposed a new structure called GF-NLFSR (Generalized Feistel-NonLinear Feedback Shift Register), and also examined the security of the structure against many attacks such as differential, linear, impossible differential and integral cryptanalysis. Furthermore, they designed a new block cipher called Four-Cell which is based on 4-cell GF-NLFSR structure. In this paper, we proved that for $n$-cell GF-NLFSR structure there exists $\left(n^{2}+n-2\right)$ rounds impossible differential. Then using this kind of 18-round impossible differential distinguisher together with some novel differential and impossible differential cryptanalysis techniques, we presented an impossible differential attack on the full 25-round Four-Cell. The data complexity of our attack is $2^{111.5}$ and the time complexity is less than $2^{123.5}$ encryptions. In addition, we expect the attack to be more efficient when the relations between different round subkeys can be exploited by taking the key scheduling algorithm into consideration.

Compared with the other kinds of generalized Feistel strucutres, the $n$-cell GF-NLFSR structure has some obvious advantage such as the ability of being parallel. However, if it is used to design a new block cipher, more work still need to be done about the security of the structure against various cryptanalysis and its pseudorandomness.

\section{References}

1. K.Nyberg and L.Kundsen, Provable Security against Differential Cryptanalysis, Journal of Cryptology, Vol.1, No.8, pp:156-168, 1995.

2. L.Knudsen, Practically secure Feistel ciphers, Fast Software Encryption- FSE'93, pp:211-221, Springer-Verlag, 1994. 
3. M.Kanda, Practical security evaluation against differential and linear attacks for Feistel ciphers with SPN round function, Selected Areas in Cryptography-SAC 2000, pp:168-179, Springer-Verlag, 2000.

4. M.Luby and C.Rackoff, How to construct pseudorandom permutations from pseudorandom functions, SIAM Journal on Computing, Vol.17, No.2, pp:373-386, 1988.

5. S.Lucks, Faster Luby-Rackoff ciphers, Fast Software Encryption-FSE'96, LNCS 1039, pp:189-203, Springer-Verlag, 1996.

6. S.Patel, Z.Ramzan, and G.Sundaram, Towards making Luby-Rackoff ciphers optimal and practical, Fast Software Encryption-FSE'99, LNCS 1636, pp:171-185, Springer-Verlag, 1999.

7. M.Naor and O.Reingold, On the construction of pseudorandom permutations Luby- Rackoff revisited, Journal of Cryptology, Vol.12, No.1, pp:9-66, 1999.

8. U.Maurer and K.Pietrzak, The security of Many-Round Luby-Rackoff Pseudorandom Permutation, Advances in Cryptology-EUROCRYPT 2003, LNCS 2656, pp:544-561, Springer-Verlag, 2003.

9. J.Patarin, Security of Random Feistel schemes with 5 or more rounds, Advances in Cryptology-CRYPTO 2004, LNCS 3152, pp:106-122, Springer-Verlag, 2004.

10. Wu Wenling, Pseudorandomness of Camellia-like scheme, Journal of Computer Science and Technology, Vol.12, No.1, pp:1-10, 2006.

11. M.Matsui, New Structure of Block Ciphers with Provable Security against Differential and Linear Cryptanalysis, Fast Software Encryption-FSE'96, LNCS 1039, pp:205-217, Springer-Verlag, 1996.

12. M.Matsui, New Block Encryption Algorithm MISTY, Fast Software EncryptionFSE'97, LNCS 1267, pp:54-68, Springer-Verlag, 1997

13. ETSI, Universal Mobile Telecommunications System (UMTS), Specification of the 3GPP confidentiality and integrity algorithms, Document 2: Kasumi specification, 2007. Available at: http://www.etsi.org/website/document/algorithms/ ts_135202v070000p.pdf.

14. T.Iwata, T.Yoshino, T.Yuasa and K.Kurosawa, Round security and super- pseudorandomness of MISTY type structure, Fast Software Encryption-FSE 2001, LNCS 2355, pp:233-247, Springer-Verlag, 2002.

15. G.Piret and J-J.Quisquater, Security of the MISTY Structure in the Luby-Rackoff Model: Improved Results, Selected Areas in Cryptography-SAC 2004, LNCS 3357, pp:100-115, Springer-Verlag, 2005.

16. Kang JS, Yi O, Hong D, et al., Pseudorandomness of Misty-type Transformations and the Block Cipher KASUMI, Australasian Conference on Information Security and Privacy-ACISP 2001, LNCS 2119, pp:60-73, Springer-Verlag, 2001.

17. T.Iwata, Tohru Yagi, and K.Kurosawa, On the Pseudorandomness of KASUMI Type Permutations, Australasian Conference on Information Security and PrivacyACISP 2003, LNCS 2727, pp:217-289, Springer-Verlag, 2003.

18. S.Vaudenay, On the Lai-Massey Scheme, Advances in Cryptology-ASIACRYPT'99, LNCS 1716, pp:9-19, Springer-Verlag, 2000.

19. P.Junod and S.Vaudenay, FOX: a new Family of Block Ciphers, Selected Areas in Cryptography-SAC 2004, LNCS 3357, pp:131-146, Springer-Verlag, 2004.

20. B.Schneier and J.Kelsey, Unbalanced Feistel Networks and Block Cipher Design, Fast Software Encryption-FSE'95, LNCS 3557, pp:121-144, Springer-Verlag, 1996.

21. C.Adams, Constructing Symmetric Ciphers Using the CAST Design Procedure, Designs, Codes and cryptography, Vol. 12, No. 3, pp:283-316, 1997.

22. MARS Block cipher http://www.nist.gov/aes/

23. Specification of SMS4, Block Cipher for WLAN Products-SMS4 (in Chinese), http://www.oscca.gov.cn/UpFile/200621016423197990.pdf 
24. T.Shirai, K.Shibutani, T.Akishita, S.Moriai, and T.Iwata, The 128-bit blockcipher CLEFIA, Fast Software Encryption-FSE 2007, LNCS 4593, pp:181-195, SpringerVerlag, 2007.

25. S.Moriai, S.Vaudenay, On the Pseudorandomness of Top-Level Schemes of Block Ciphers, Advances in Cryptology-ASIACRYPT 2000, LNCS 1976, pp: 289-302, Springer-Verlag, 2000.

26. K.Nyberg, Generlized Feistel networks, Advances in Cryptology-ASIACRYPT'96, LNCS 1163, pp:91- 104, Springer-Verlag, 1996.

27. Wenling $\mathrm{Wu}$, Wentao Zhang, and Dongdai Lin, On the Security of Generalized Feistel Scheme with SP Round Function, International Journal network security, Vol. 2, No.3, pp:296-305, 2006.

28. T.Shirai and K.Shibutani, On Feistel structures using a diffusion switching mechanism, Fast Software Encryption-FSE 2006, LNCS 4047, pp:41-56, Springer-Verlag, 2006.

29. Jiali Choy, Guanhan Chew, Khoongming Khoo and Huihui Yap, Cryptographic Properties and Application of a Generalized Unbalanced Feistel Network Structure, Australasian Conference on Information Security and Privacy-ACISP 2009, http: //eprint.iacr.org/2009/178

30. E.Biham, A.Biryukov, and A.Shamir, Cryptanalysis of Skipjack Reduced to 31 Rounds Using Impossible Differentials, Advances in Cryptology-EUROCRYPT'99, LNCS 2595, pp:12-23, Springer-Verlag, 1999.

31. Raphael Chung-Wei Phan, Impossible Differential Cryptanalysis of 7-round AES, Information Processing Letters, Vol.91, Number 1, pp:33-38, 2004.

32. Wentao Zhang, Wenling Wu, and Dengguo Feng, New Results on Impossible Differential Cryptanalysis of Reduced AES, Information Security and Cryptology-ICISC 2007, LNCS 4817, pp:239-250, Springer-Verlag, 2007.

33. Wenling $\mathrm{Wu}$, Wentao Zhang, and Dengguo Feng, Impossible Differential Cryptanalysis of Reduced-Round ARIA and Camellia, Journal of Computer Science and Technology, Vol.22, No.3, pp:449-456, 2007.

34. Y.Tsunoo, E.Tsujihara, T.Saito, T.Suzaki, and H.Kubo, Impossible Differential Cryptanalysis of CLEFIA, Fast Software Encrption-FSE 2008, LNCS 5086, pp:398411, Springer-Verlag, 2008.

35. Orr Dunkelman and Nathan Keller, An Improved Impossible Differential Attack on MISTY1, Advances in Cryptology-ASIACRYPT 2008, LNCS 5350, pp:441-454, Springer-Verlag, 2008.

36. J.Kim, S.Hong, J.Sung, S.Lee, J.Lim and S.Sung, Impossible Differential Cryptanalysis for Block Cipher Structures, Progress in Cryptology-INDOCRYPT 2003, LNCS 2904, pp:82-96, Springer-Verlag, 2003.

\section{Appendix A: Figure of Attack Procedure}




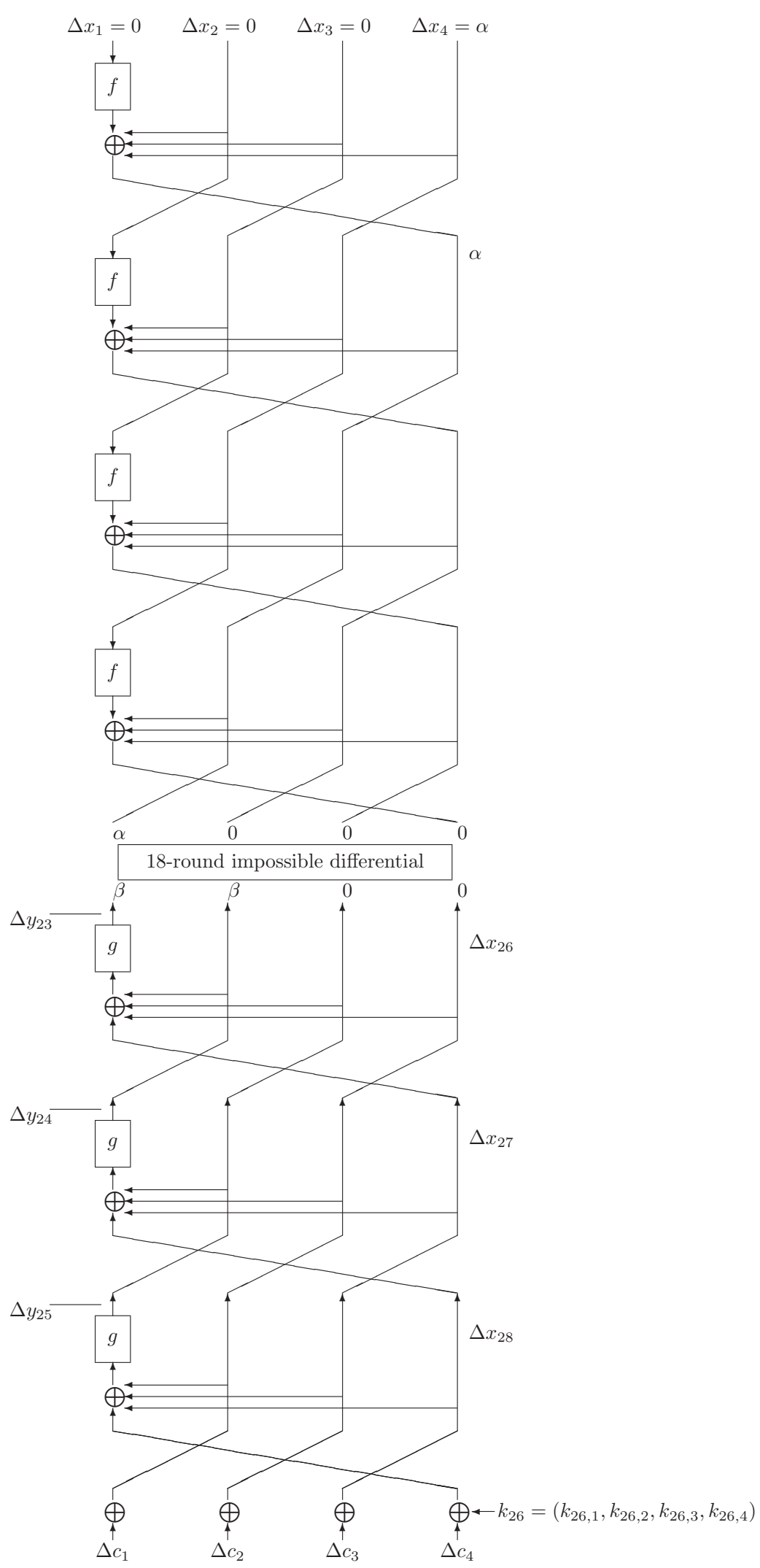

Fig. 2. Impossible Differential Attack on the Full 25-Round of Four-Cell 